\title{
Hysterosalpingo-Foam Sonography to Replace HSG in Evaluating Tubal Patency in Indonesian Subfertile Patients
}

\author{
Herbert Situmorang1,2, Harry Prawiro Tantry1,2, Eka Rusdianto Gunardi ${ }^{1,2}$ \\ ${ }^{1}$ Department of Obstetrics and Gynecology, Faculty of Medicine, University of Indonesia, Jakarta, Indonesia \\ ${ }^{2}$ Department of Obstetrics and Gynecology, Dr. Cipto Mangunkusumo National Central General Hospital, Jakarta, Indonesia \\ Email: herbert.situmorang@ui.ac.id
}

How to cite this paper: Situmorang, $\mathrm{H}$., Tantry, H.P. and Gunardi, E.R. (2020) Hysterosalpingo-Foam Sonography to Replace HSG in Evaluating Tubal Patency in Indonesian Subfertile Patients. Open Journal of Obstetrics and Gynecology, 10, 1402-1413

https://doi.org/10.4236/ojog.2020.10100129

Received: August 17, 2020

Accepted: October 13, 2020

Published: October 16, 2020

Copyright $\odot 2020$ by author(s) and Scientific Research Publishing Inc. This work is licensed under the Creative Commons Attribution International License (CC BY 4.0).

http://creativecommons.org/licenses/by/4.0/

\begin{abstract}
Aim: To evaluate whether hysterosalpingo-foam sonography (HyFoSy) can replace HSG as first-line evaluation for tubal patency in subfertile Indonesian patients by evaluating agreement level and comparing patients' subjective complaints. Methods: Twenty subfertile female patients at Cipto Mangunkusumo Central General Referral Hospital (RSCM) from January 2018 to January 2019 were recruited conveniently to undergo both HSG and subsequent two-dimensional transvaginal HyFoSy after a minimum interval of 48 hours. Data on tubal patency and side effects inflicted by each examination within 24 hours were collected. Results: There were 20 subjects enrolled in this study. In 2 subjects, stenosis of internal uterine ostium was identified on HyFoSy procedure. Out of 36 remaining tubes undergoing adequate tubal patency evaluation by HSG and HyFoSy, agreement was seen in $81 \%$ cases (kappa value 0.42). The discordant tubal evaluation results in 7 of 36 tubes were associated with partial tubal obstruction, presence of co-existing gynaecological pathology, and tubal spasm. Less pain $(\mathrm{p}<0.001)$ was experienced in HyFoSy as compared to HSG, with mean VAS of $1.8 \pm 1.4 \mathrm{~cm}$ and $5.4 \pm 2.4$ $\mathrm{cm}$, respectively. Seven of 20 patients experienced side effects due to HSG, such as abdominal cramp, spotting, backache, and bloating, in contrast to only one subject experiencing spotting due to HyFoSy. There was no hypersensitivity reaction observed in all subjects during HSG and HyFoSy procedure. Conclusions: HyFoSy has shown an excellent acceptance in Indonesian patients and could be a good alternative to replace HSG as a first line tubal patency examination.
\end{abstract}

\section{Keywords}

HyFoSy, HSG, Tubal Patency, Subfertile 


\section{Introduction}

Approximately $15 \%$ of couples suffered from subfertility, $40 \%$ of which were attributed to tubal factor in developing countries [1] [2] necessitating a feasible and well-tolerated tubal patency examination across the geographically scattered islands in Indonesia for easier and earlier access to subfertility treatment. An emerging non-invasive and bedside tubal patency examination with superior diagnostic value and patient's satisfaction as compared to HSG, known as hysterosalpingo-foam sonography (HyFoSy), has been initiated in Indonesia at Ciptomangunkusumo Central General Referral Hospital (RSCM) since 2018. Ludwin et al. described that HyFoSy has a sensitivity of $87 \%$ and specificity of $94 \%$ [3], which was higher than HSG that had a sensitivity of $63 \%$ and specificity of 89\% [4]. Zizolfi et al. in Italy performed a concordance study between $\mathrm{HyFOSy}$ and HSG that was performed on 50 women who had undergone hysteroscopic Essure microinsert placement and found a concordance rate of 100\% [5]. Another study by Dreyer et al. in the Netherlands showed a $97.4 \%$ concordance between HyFoSy and HSG in evaluating tubal occlusion in 26 women undergoing Essure placement due to hydrosalpinx before in vitro fertilization [6].

Standard HSG examination for tubal patency has several drawbacks in patient's perspective, among others is pain during and after procedure, allergy to contrast agent, side effects, and the lengthy procedure of arranging appointment to the radiology department and returning the HSG result to the gynecologist on the multiple visits. Meanwhile, HyFoSy offers an instant tubal patency result in an approximately 5-minute bedside procedure requiring only a two-dimensional (2D) transvaginal ultrasonography and the ExEm-foam kit consisting of hydroxyethylcellulose-glycerol gel and its cervical cannula. Tolerance study that was performed by Dreyer et al. in 2015 in the Netherlands to compare the pain experienced during HyFoSy in comparison to HSG found a significantly lower pain score for HyFoSy with median VAS score of $2.0 \mathrm{~cm}$ compared to HSG with median VAS score of $5.1 \mathrm{~cm} \mathrm{[6].}$

To the best of our knowledge, this study was the first implementation of $\mathrm{Hy}$ FoSy in Indonesian population. Although HyFoSy had shown an excellent acceptance in Caucasian patients [6] [7] [8], there was yet a tolerance study on Indonesian population. Considering the possible effect of ethnicity on patients' tolerance, as already exemplified on the frequency of carbamazepine-induced Steven-Johnson syndrome/toxic epidermal necrolysis that was only 5\% - 6\% in Europe in contrast to $25 \%$ - 33\% in Taiwan [9] [10], therefore this study on Indonesian population was necessary to prove the safety and acceptability of HyFoSy procedure in Indonesian patients. The aim of this study was to evaluate whether HyFoSy can replace HSG as a first-line evaluation for tubal patency in subfertile Indonesian patients by evaluating their agreement rate and patients' tolerance level.

\section{Methods}

This concordance study was conducted prospectively in RSCM from January 
2018 to January 2019. Inclusion criteria were all subfertile patients with stable hemodynamic at the outpatient clinic, while exclusion criteria were patients with cervical neoplasm, Mullerian agenesis, and impaired kidney function. A total of 20 subfertile Indonesian women were recruited conveniently and underwent both HSG and subsequent HyFoSy after a minimum interval of 48 hours. Data on side effects inflicted by each examination within 24 hours was collected by interview. The secondary outcome measures included pain experienced during HyFoSy and HSG, measured by Visual Analogue Scale (VAS) scores (1 - $10 \mathrm{~cm}$ ), as well as nominal data on subjective complaints experienced by the subjects within 24 hours since the initiation of the procedure.

While the HSG procedures were performed in one appointed radiology installation with a standard procedure of passing radio-opaque dye from the cervical canal to the uterine cavity under fluoroscopic guidance that was then interpreted by the radiologist on duty, all the HyFoSy procedures were performed by a single gynaecologist in outpatient setting. The HyFoSy procedure was performed in lithotomy position. The foam was prepared by mixing $10 \mathrm{ml}$ of hydroxyethylcellulose-glycerol gel with $10 \mathrm{ml}$ of purified water until it turned white (Figure 1). A speculum was inserted to the vagina to visualize the cervix. Then, a flexible cervical balloon-less cannula provided by the ExEm foam kit was inserted to the cervix to provide a path for foam infusion to the uterine cavity. Following removal of the speculum, the transvaginal ultrasound transducer was inserted into the vagina to visualise the longitudinal plane of the uterus, while the other hand of the operator pushed the plunger of the syringe gently to introduce the foam into the uterine cavity. After the foam was identified as a hyperechoic line flowing within the uterine cavity in the longitudinal plane, the transducer was rotated to the transverse plane to locate the ovary and the transducer was adjusted to visualise the hyperechoic foam from the uterine cavity that

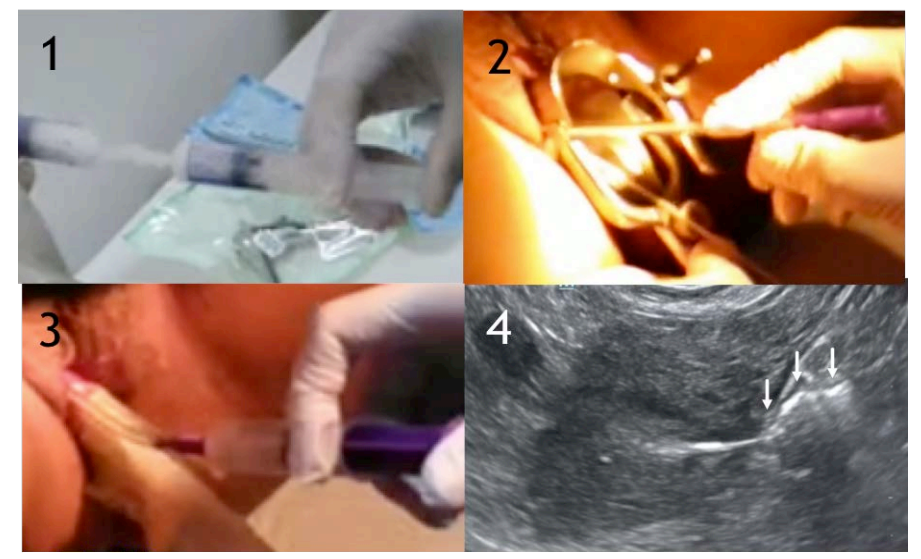

Figure 1. The foam used in HyFoSy was created by mixing $10 \mathrm{ml}$ of hydroxyethylcellulose-glycerol gel with $10 \mathrm{ml}$ of purified water until it turned white (1). Speculum was used to visualise the cervix and cervical cannula was inserted into the cervical ostium (2). The foam was infused through the cervical cannula under transvaginal ultrasonography guidance (3). The flow of hyperechoic foam (white arrow) along the lumen of Fallopian tube indicated tubal patency (4). 
continued to flow along the fallopian tube and end as dispersion to the peritoneal cavity. Similar step was performed on the contralateral side to evaluate the patency of the contralateral Fallopian tube. To preclude operator bias, all HyFoSy procedures were performed by a single operator with adequate training from the representative of the ExEm foam kit manufacturer. The study protocol was approved by the Ethics Committee of the Faculty of Medicine University of Indonesia on December 29 $9^{\text {th }}, 2017$ (reference number: 1163/UN2.F1/ETIK/2017). All participating women gave informed consent.

Statistical analyses were conducted to assess the agreement rate of tubal patency results between HyFoSy as the index test and HSG as the reference standard by measuring the Kappa Cohen value. The primary outcome of accuracy was calculated by adding the sum of true positives and true negatives, and then divided by the sum of true positives, true negatives, false positives and false negatives. Secondary outcome of numerical pain score between HyFoSy and HSG was analysed using paired t-test. A p-value of $<0.05$ indicated a statistically significant difference. Statistical analyses were performed using the IBM Statistical Package for Social Sciences (SPSS) version 23.0 (IBM Corp., USA). Other nominal data of subjective complaints within 24 hours after the initiation of HyFoSy and HSG was presented with descriptive statistics.

\section{Results}

Between January 2018 and January 2019, twenty subfertile Indonesian females were examined with HSG and subsequent HyFoSy to demonstrate tubal patency. The mean age of patients was $33.5 \pm 6.0$ years with a mean subfertility duration of $4.0 \pm 3.6$ years. Of the 20 included subjects, one patient was categorised as secondary subfertility and five patients admitted dysmenorrhea (Table 1).

All women underwent HSG followed by HyFoSy after a minimum interval of 48 hours. Of the 20 women having undergone adequate tubal evaluation by HSG, 2 women showed internal uterine ostium stenosis during HyFoSy that voided tubal patency evaluation from these $2 \mathrm{HyFoSy}$ procedures (Figure 2). Hence, only 36 of 40 tubes were included in the agreement analysis.

Table 1. Demographic and clinical characteristics.

\begin{tabular}{cc} 
Variable & $\begin{array}{c}\text { Description } \\
(\mathrm{n}=20)\end{array}$ \\
\hline Age (mean \pm standard deviation) & $33.5 \pm 6.0$ year \\
Subfertility duration (mean \pm standard deviation) & $4.0 \pm 3.6$ year \\
Primary subfertility (n, \%) & $19 / 20,95 \%$ \\
Secondary subfertility (n, \%) & $1 / 20,5 \%$ \\
Dysmenorrhea $(\mathrm{n}, \%)$ & $5 / 20,25 \%$ \\
\hline
\end{tabular}


From the 36 tubes that had undergone adequate tubal patency evaluation by HSG and HyFoSy, agreement was seen in $81 \%$ of cases with a moderate kappa value of 0.42 . During HSG, 30 of 36 tubes were described as patent, while 6 of 36 tubes were non-patent. In comparison, 27 of 36 tubes were visualised as patent during HyFoSy, whereas 9 of 36 were occluded (Table 2).

Discrepancy of tubal patency results between HSG and HyFoSy was seen in 7 of 36 tubes. Five of which were patent according to HSG, but the tubal patency result during HyFoSy described otherwise. Meanwhile, 2 tubes were showing occlusion during HSG, but were found to be patent during HyFoSy examination.

Patients were asked to rate the worst pain experienced within 24 hours following HSG and HyFoSy procedure using the VAS score, with 0 corresponding to no pain and 10 corresponding to maximum pain. The VAS score was significantly lower in HyFoSy compared to HSG, with mean VAS score of $1.8 \pm 1.4$ and $5.4 \pm 2.4(\mathrm{p}<0.001)$, respectively (Table 3$)$.

Seven out of 20 patients experienced mild side effects during HSG, such as abdominal cramp, spotting, backache, and bloating, in contrast to only 1 patient experiencing spotting in the HyFoSy group (Figure 3). Subjective complaints during HSG were in the form of spotting ( 7 of 20 patients), abdominal cramp (7 of 20 patients), backache ( 2 of 20 patients), and bloating ( 1 of 20 patients). None of subjects in this study experienced hypersensitivity reaction or severe adverse effects due to HSG or HyFoSy procedure.

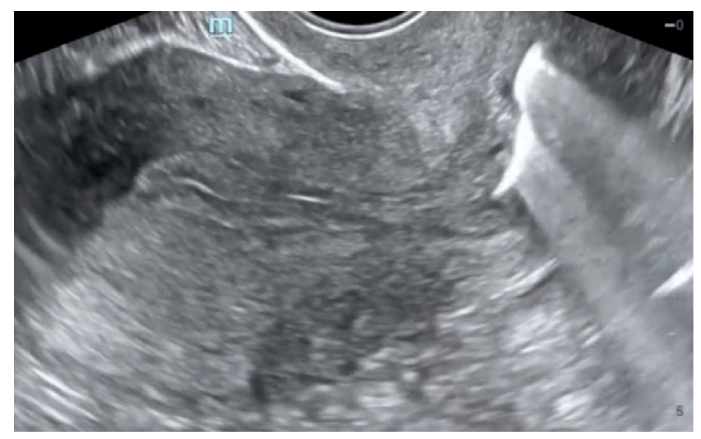

Figure 2. The flow of hyperechoic foam seen in the longitudinal plane was stopped at the internal uterine ostium due to stenosis in 2 of 20 subjects during HyFoSy procedures.

Table 2. Primary outcome of tubal patency according to HyFoSy and HSG.

\begin{tabular}{ccc}
\hline Evaluation & $\begin{array}{c}\text { Patent tube in HSG } \\
(\mathrm{n}=30)\end{array}$ & $\begin{array}{c}\text { Non-patent tube in HSG } \\
(\mathrm{n}=6)\end{array}$ \\
\hline Patent tube in $\mathrm{HyFoSy}_{\mathrm{f}}(\mathrm{n}=27)$ & 25 & 2 \\
Non-patent tube in $\mathrm{HyFOSy}_{\mathrm{F}}(\mathrm{n}=9)$ & 5 & 4 \\
\hline
\end{tabular}

Table 3. Pain experienced within 24 hours after HyFoSy and HSG.

\begin{tabular}{cccc}
\hline Pain & HyFoSy & HSG & p value \\
\hline VAS score & $1.8 \pm 1.4$ & $5.4 \pm 2.4$ & $<0.001$ \\
\hline
\end{tabular}




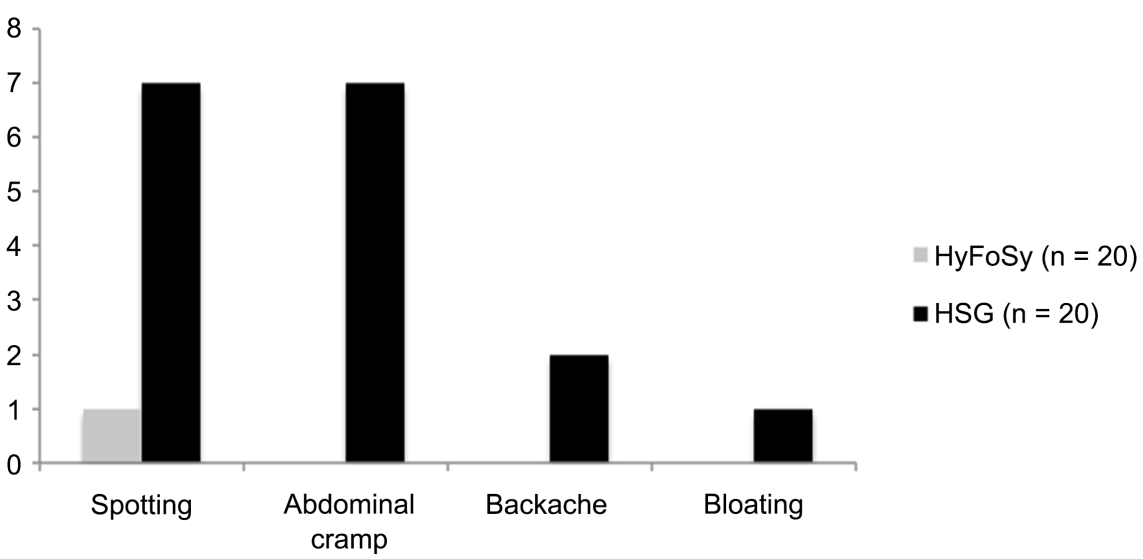

Figure 3. Less side effects were experienced during HyFoSy compared to HSG. Seven of 20 patients experienced side effects due to HSG, such as abdominal cramp, spotting, backache, and bloating, in contrast to only one subject experiencing spotting due to HyFoSy.

\section{Discussion}

To the best of our knowledge, this is the first study in Indonesian population evaluating the agreement level between HyFoSy and HSG in evaluating tubal patency. Our result showed a moderate agreement level of $81 \%$ (Cohen's kappa coefficient level of 0.42), which was lower than previous studies by Zizolfi et al. in Italy with $100 \%$ agreement [5] and by Dreyer et al. in the Netherlands with 97.4\% agreement [6]. This difference was likely due to different study population since the subjects in these two studies were women with Essure placement that occluded the proximal part of the Fallopian tubes. Meanwhile, subjects in this study were subfertile patients with no Essure placement that was comprised of only $20 \%$ occluded tubes according to HyFoSy or $17 \%$ occluded tubes according to HSG. Therefore, it could be implied that HyFoSy had a better agreement with HSG in cases of tubal occlusions.

The agreement level in this study was also affected by the foundational difference of accuracy between HSG and HyFoSy when compared with the gold standard laparoscopy chromotubation. HSG had been described to have a sensitivity level of $63 \%$ and specificity of $89 \%$ [4], while HyFoSy had a higher sensitivity of $87 \%$ and specificity of $94 \%$ [3]. An unrandomized observational study of 20 women by Van Schoubroeck et al. even found $100 \%$ agreement in tubal assessment results between laparoscopy chromotubation and HyFoSy [11]. This study did not measure the accuracy of HyFoSy by comparing it with the gold standard tubal patency examination that was laparoscopy chromotubation because the first line tubal patency evaluation used in Indonesia was HSG. Therefore, a moderate agreement between HyFoSy and HSG in this study was sufficient to validate the implementation of HyFoSy as an alternative to HSG as a first line tubal patency examination in Indonesia.

Interestingly, this study found discordant tubal evaluation results in 7 of 36 tubes. In 3 of 5 tubes with occlusion according to HyFoSy yet patent according 
to HSG, there was a need to increase the pressure of the contrast infusion during HSG that only manage to create little spill to the peritoneal cavity that did not spread well. That indicated that there were partial obstructions within those three tubes that could be classified as patent but diseased tubes. According to a review article by Patil in 2009, results of tubal patency evaluation by HSG could be classified as normal tube, patent tube with tubal disease, and blocked tubal disease. In his comparison, Patil described the normal tubes to have good prognosis for future fertility with very low incidence of ectopic pregnancy. In contrast, blocked tubes had poor prognosis for non-assisted future fertility with very low incidence of ectopic pregnancy. Meanwhile, patent tubes with tubal disease had the most hostile prognosis since it had a moderate fertility prognosis with the highest incidence of ectopic pregnancy of up to $25 \%$ - 50\% [12] [13]. According to the findings of this study, these patent tubes with tubal disease could be distinguished by HSG examination while the HyFoSy with lower infusion pressure may group these diseased tubes altogether with the completely blocked tubes.

Another cause of patent tube according to HSG that was shown occluded by HyFoSy was obstruction by extra tubal mass. In Figure 4, the presence of adenomyosis obstructed the flow of foam through the interstitial part of the Fallopian tube during HyFoSy, yet the higher contrast infusion pressure in HSG could overcame the resistance caused by the extra tubal adenomyosis and created peritoneal spill that was interpreted as patent tube while overlooking the presence of the radiolucent adenomyosis. Considering that the physiologic transport of gametes within the fallopian tube was not augmented by external pressure [14], therefore the patency described by the HSG in this case could be regarded as a false negative result. Standing on another point of view, HSG could be a testing modality to predict whether removal of an adjacent obstructing mass would return the patency of an obstructed tube.

In this study, the 2 cases of occluded tubes according to HSG that were shown patent by HyFoSy comprised of 1 proximal occlusion and 1 distal occlusion. Proximal tubal occlusion had been previously described to have a false positive rate of $39 \%$ due to tubal spasm [15]. Therefore, the case of proximal tubal occlusion, seen as the absence of contrast filling beyond the interstitial part during HSG (Figure 5) that was seen patent in HyFoSy, was likely due to tubal spasm.

Meanwhile, the case of distal occlusion observed in the discordant case was caused by the displacement of the Fallopian tube to the lateral side due to an undetected radiolucent large leiomyoma, hence no contrast spill was seen spreading to the pelvic floor during HSG examination. Interestingly, the leiomyoma could be clearly seen during HyFoSy procedure and the hyperechoic foam could be tracked flowing from the uterine cavity, passing through the interstitial part of the Fallopian tube, and filling the whole Fallopian tube along the perimeter of the leiomyoma until it spilled to the peritoneal cavity (Figure 6). 


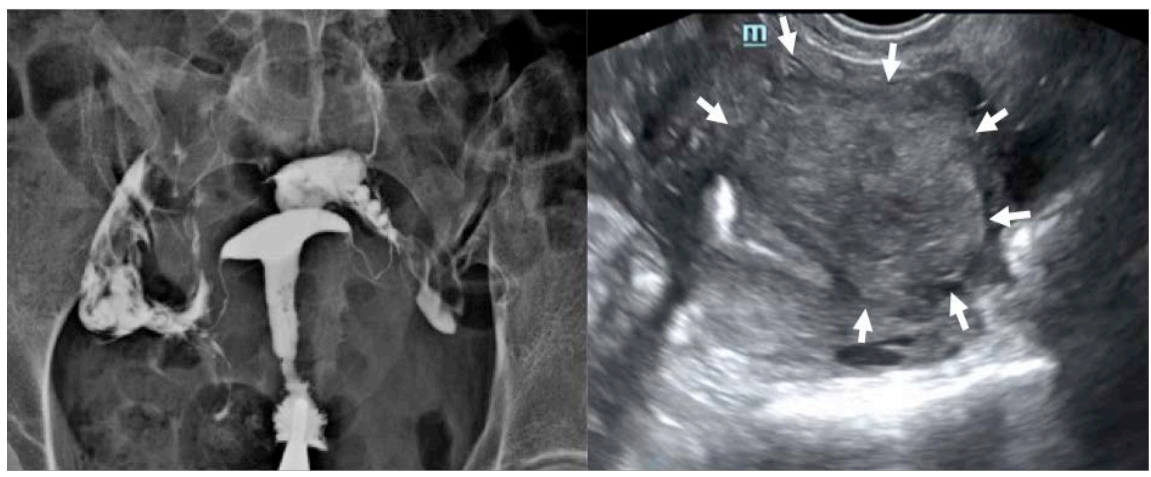

Figure 4. An adenomyosis (white arrows) obstructed the flow of foam through the interstitial part of the left Fallopian tube during HyFoSy (right). In the same tube, the higher contrast infusion pressure in HSG could overcome the resistance caused by the extra tubal obstruction and created peritoneal spill that was interpreted as patent left tube while overlooking the presence of the radiolucent adenomyosis (left).

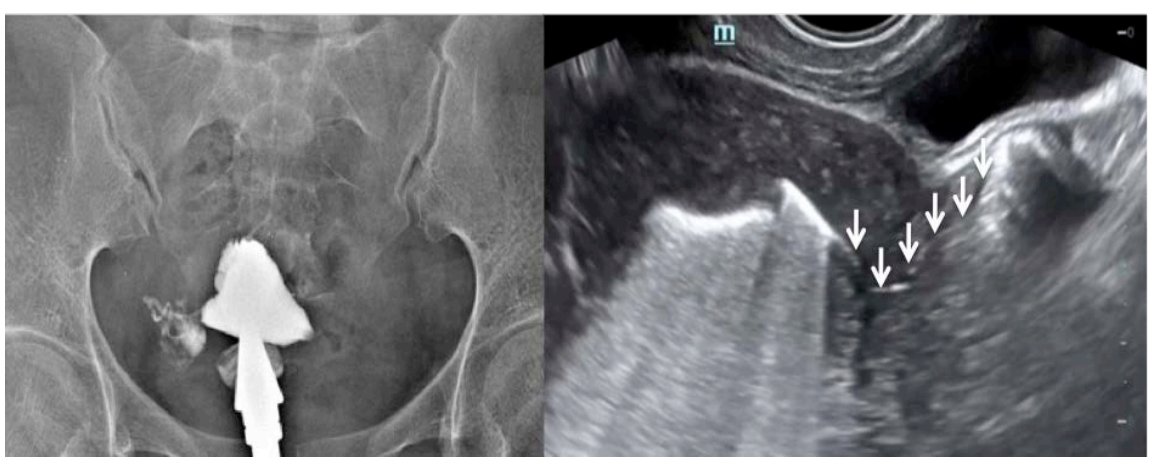

Figure 5. A case of left proximal tubal occlusion, seen as the absence of contrast filling beyond the interstitial part during HSG (left), had a 39\% probability of false positive due to tubal spasm. During HyFoSy, hyperechoic foam (white arrows) was visualised flowing seamlessly from the uterine cavity through the interstitial part until the whole course of the same Fallopian tube (right).

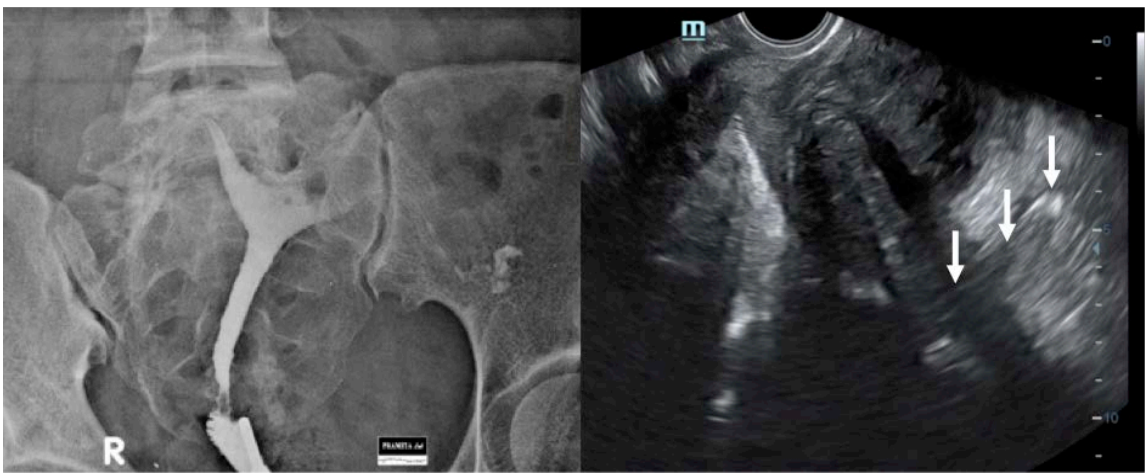

Figure 6. The left Fallopian tube was displaced to the lateral side due to an undetected radiolucent large leiomyoma, hence no contrast spill was seen spreading to the left pelvic floor during HSG examination (left). In the same Fallopian tube, the adjacent leiomyoma could be clearly seen during HyFoSy procedure and the hyperechoic foam (white arrows) could be tracked flowing from the uterine cavity, passing through the interstitial part of the Fallopian tube, and filling the whole Fallopian tube along the perimeter of the leiomyoma until it spilled to the peritoneal cavity. 
Another utility of HyFoSy found during this study was to detect stenosis of internal uterine ostium. From 20 subjects with adequate evaluation of tubal status by HSG, 2 of them could not have tubal status evaluation by HyFoSy since the foam could not pass beyond the internal cervical ostium that corresponded with stenosis of internal uterine ostium (Figure 2). Cases of stenosis hindering tubal evaluation by HyFoSy had been previously described by Emanuel et al. From 73 subjects to whom Emanuel et al. performed HyFoSy, 6 subjects had unsuccessful HyFoSy procedure due to cervical blockage in 5 patients and leakage in 1 patient [16]. During HyFoSy procedure, the foam could not pass the narrowed internal uterine ostium because the type of cervical cannula provided in the ExEm foam kit was flexible cannula and was not assisted by tenaculum. In comparison, HSG procedure employed a metallic cervical cannula that was fixated by tenaculum. Therefore, the cervical cannula in HyFoSy would bend instead of forcing through upon meeting resistance. In contrast, the metallic cervical cannula used in HSG may exert blunt force that, though more traumatic, may provide benefit by mechanically dilating a pre-existing stenosis of internal uterine ostium.

Considering that HyFoSy had similar procedure to HSG in terms of creating distension of the uterine cavity, therefore the anticipated side effects due to both procedures would both encompass possibility of vasovagal reaction and pain. This study found a significantly less pain $(\mathrm{p}<0.001)$ due to HyFoSy with VAS score of $1.8 \pm 1.4 \mathrm{~cm}$ in comparison to HSG with VAS score of $5.4 \pm 2.4 \mathrm{~cm}$. More proportion of subjects complained side effects in the form of spotting, abdominal cramp, backache, and bloating due to HSG in comparison to HyFoSy. Within 24 hours since the initiation of HSG in 20 subjects, 35\% of subjects experienced spotting, $35 \%$ of subjects experienced abdominal cramp, $10 \%$ of subjects experienced backache, and 5\% of subjects experienced bloating. In contrast, apart from pain, spotting was the only side effect complained due to HyFoSy and was only found in $5 \%$ of subjects. These findings were consistent with previous studies in Europe that had shown that HyFoSy had a better patient's tolerance compared to HSG. Randomized controlled trial by Dreyer et al. found that HyFoSy was statistically less painful $(\mathrm{p}<0.01)$ with a median VAS score of $1.7 \mathrm{~cm}$ in comparison to HSG with a median VAS score of $3.7 \mathrm{~cm}$ [8]. There was no hypersensitivity reaction or severe adverse effect observed in 20 Indonesian women included in this study following HyFoSy or HSG procedure. Likewise, study by Schoubroeck et al. in 216 patients undergoing HyFoSy found that 92.1\% of subjects regarded the worst side effect due to HyFoSy was only a tolerable pain [7].

In regard to possible effect of pain scores with the patient knowing what to expect in the second investigation, study by Sipila et al. had shown that expectation of severe pain was associated with higher scores of both experimental and clinical pain intensity. Therefore the higher VAS score in the HSG procedure as compared to the subsequent HyFoSy procedure in this study was not biased by 
psychological factor of expecting pain in the subsequent procedure [17]. In regard to possible therapeutic effect of conventional HSG that may have altered the findings on HyFoSy, the studies on the effect of tubal flushing with HSG to increase live birth rate had a low quality of evidence [18]. Therefore, conventional HSG was not likely to have altered the findings on HyFoSy in our study.

Chou Phay Lim et al. argued that HSG was out of date and had no place in modern infertility work up in the emergence of newer tubal patency examinations [19]. As seen in this study, HyFoSy had better patient's tolerance with moderate agreement to HSG while avoiding radiation exposure, omitting the need of fluoroscopy instrument and shortening the diagnostic time by deducting liaison with radiology department. Yet, this study had also shown that HSG may be a better option to evaluate tubal patency in the presence of stenosis of internal uterine ostium. HSG also allowed comparison of contrast spill with the contralateral side in conjunction with the difference in contrast infusion pressure needed that would be useful to distinguish patent but diseased tubes with high risk for ectopic pregnancy. Furthermore, HyFoSy could offer a wider utility beyond dichotomizing between a patent tube and an occluded tube. As seen from this study, when HyFoSy was complemented with the higher contrast infusion pressure of HSG, it could distinguish a candidate that might benefit from removal of obstructing extra tubal mass.

A challenge in the dissemination of the practice of $2 \mathrm{D}-\mathrm{HyF}$ oSy in Indonesia would be the necessity to climb the learning curve. Ranaweera et al. had suggested a shallow learning curve for a person with experience in transvaginal ultrasound to achieve confidence in performing saline sonographic hydrotubation after 5 - 10 cases [20]. Considering that the foam in HyFoSy can visualise Fallopian tube far longer than saline, therefore a similar learning curve threshold of 5 to 10 cases would be adequate to achieve confidence in performing HyFoSy.

\section{Conclusion}

HyFoSy had a moderate agreement of $81 \%$ (kappa value of 0.42 ) to HSG and had a better patient's tolerance as compared to HSG in Indonesian population. Hence, HyFoSy is a potentially more preferable alternative as a first line tubal patency evaluation in subfertile Indonesian patients.

\section{Acknowledgements}

The authors thank Cipto Mangunkusumo National Central General Hospital in Indonesia for fully funding this research through Operational Research Grant 2018.

\section{Conflicts of Interest}

The authors declare no conflicts of interest regarding the publication of this paper.

\section{References}

[1] Dun, E.C. and Nezhat, C.H. (2012) Tubal Factor Infertility. Diagnosis and Man- 
agement in the Era of Assisted Reproductive Technology. Obstetrics and Gynecology Clinics of North America, 39, 551-566. https://doi.org/10.1016/j.ogc.2012.09.006

[2] Ombelet, W., Cooke, I., Dyer, S., Serour, G. and Devroey, P. (2008) Infertility and the Provision of Infertility Medical Services in Developing Countries. Human Reproduction Update, 14, 605-621. https://doi.org/10.1093/humupd/dmn042

[3] Ludwin, I., Ludwin, A., Wiechec, M., Nocun, A., Banas, T., Basta, P., et al. (2017) Accuracy of Hysterosalpingo-Foam Sonography in Comparison to Hysterosalpingo-Contrast Sonography with Air/Saline and to Laparoscopy with Dye. Human Reproduction, 32, 758-769. https://doi.org/10.1093/humrep/dex013

[4] Sakar, M.N., Gul, T., Atay, A.E. and Celik, Y. (2008) Comparison of Hysterosalpingography and Laparoscopy in the Evaluation of Infertile Women. Saudi Medical Journal, 29, 1315-1318.

[5] Zizolfi, B., Lazzeri, L., Franchini, M., Sardo, A.D.S., Nappi, C., Piccione, E., et al. (2018) One-Step Transvaginal Three-Dimensional Hysterosalpingo-Foam Sonography (3D-HyFoSy) Confirmation Test for Essure ${ }^{\circledast}$ Follow-Up: A Multicenter Study. Ultrasound in Obstetrics \& Gynecology, 51, 134-141.

https://doi.org/10.1002/uog.17398

[6] Dreyer, K., Hompes, P.G.A. and Mijatovic, V. (2015) Diagnostic Accuracy of Hysterosalpingo-Foam Sonography to Confirm Tubal Occlusion after Essure ${ }^{\circledast}$ Placement as Treatment for Hydrosalpinges. Reproductive BioMedicine Online, 30, 421-425. https://doi.org/10.1016/j.rbmo.2014.12.009

[7] Van Schoubroeck, D., Van den Bosch, T., Ameye, L., Boes, A.S., D’Hooghe, T. and Timmerman, D. (2015) Pain during Fallopian-Tube Patency Testing by Hysterosalpingo-Foam Sonography. Ultrasound in Obstetrics \& Gynecology, 45, 346-350. https://doi.org/10.1002/uog.14646

[8] Dreyer, K., Out, R., Hompes, P.G. and Mijatovic, V. (2014) Hysterosalpingo-Foam Sonography, a Less Painful Procedure for Tubal Patency Testing during Fertility Workup Compared with (Serial) Hysterosalpingography: A Randomized Controlled Trial. Fertility and Sterility, 102, 821-825. https://doi.org/10.1016/j.fertnstert.2014.05.042

[9] Chung, H.W., Hung, S.L., Hong, H.S., Hsih, M.S., Yang, L.C., Ho, H.C., et al. (2004) A Marker for Stevens-Johnson Syndrome. Nature, 428, 486. https://doi.org/10.1038/428486a

[10] Shah, R.R. and Gaedigk, A. (2018) Precision Medicine: Does Ethnicity Information Complement Genotype-Based Prescribing Decisions? Therapeutic Advances in Drug Safety, 9, 45-62. https://doi.org/10.1177/2042098617743393

[11] Van Schoubroeck, D., Van den Bosch, T., Meuleman, C., Tomassetti, C., D’Hooghe, T. and Timmerman, D. (2013) The Use of a New Gel Foam for the Evaluation of Tubal Patency. Gynecologic and Obstetric Investigation, 75, 152-156. https://doi.org/10.1159/000345865

[12] Patil, M. (2009) Assessing Tubal Damage. Journal of Human Reproductive Sciences, 2, 1-11. https://doi.org/10.4103/0974-1208.51335

[13] Patil, E. and Thurmond, A. (2015) The History and Current Status of Fallopian Tube Pressures-Developing Alternate Methods for Confirmation of Tubal Occlusion. Contraception, 92, 124-127.

https://doi.org/10.1016/j.contraception.2015.01.003

[14] Croxatto, H.B. (2002) Physiology of Gamete and Embryo Transport through the Fallopian Tube. Reproductive BioMedicine Online, 4, 160-169. 
https://doi.org/10.1016/S1472-6483(10)61935-9

[15] Schankath, A.C., Fasching, N., Urech-Ruh, C., Hohl, M.K. and Kubik-Huch, R.A. (2012) Hysterosalpingography in the Workup of Female Infertility: Indications, Technique and Diagnostic Findings. Insights Imaging, 3, 475-483. https://doi.org/10.1007/s13244-012-0183-y

[16] Emanuel, M.H., Van Vliet, M., Weber, M. and Exalto, N. (2012) First Experiences with Hysterosalpingo-Foam Sonography (HyFoSy) for Office Tubal Patency Testing. Human Reproduction, 27, 114-117. https://doi.org/10.1093/humrep/der367

[17] Sipila, R.M., Lassi, H., Maretoja, T.J., Ripatti, S., Estlander, A.M. and Kalso, E.A (2017) Does Expecting Pain Make It More Intense? Factors Associated with the First Week Pain Trajectories after Breast Cancer Surgery. Pain, 158, 922-930. https://doi.org/10.1097/j.pain.0000000000000859

[18] Van Rijswijk, J., Van Welie, N., Dreyer, K., Tajik, P., Lambalk, C.B., Hompes, P., et al. (2019) Tubal Flushing with Oil- or Water-Based Contrast Medium: Can We Identify Markers That Indicate Treatment Benefit? Human Reproduction Open, 2019, hoz015. https://doi.org/10.1093/hropen/hoz015

[19] Lim, C.P., Hasafa, Z., Bhattacharya, S. and Maheswari, A. (2011) Should a Hysterosalpingogram Be a First-Line Investigation to Diagnose Female Tubal Subfertility in the Modern Subfertility Workup? Human Reproduction, 26, 967-971.

https://doi.org/10.1093/humrep/der046

[20] Ranaweera, A.K.P., Batcha, M. and Kalansooriya, H. (2013) Value of Sonographic Hydrotubation Using Agitated Saline as a Screening Test for Tubal Patency. SJAMS, $1,122-130$. 